ly. This is, however, only an apparent cross section. The true capture cross section $\sigma_{p}^{\prime}$ is larger and is related to $\sigma_{p}$ by

$$
\sigma_{p}^{\prime}=\sigma_{p} \exp \left(\phi_{b} / k T\right),
$$

because the carriers have to overcome the grain-boundary potential $\phi_{B}$ to be captured. From these measurements it was also found that for saturating pulse widths the concentration of trap $H_{1}$ was $\sim 10^{i 4} \mathrm{~cm}^{-3}$.

DLTS signals of hole traps in comparable material to Fig. 1(a) obtained with mesa $n^{+} p$ structures looked very similar to those of Fig. 1, indicating the presence of the same traps. Peak $H_{4}$, however, was less prominent, probably a result of the cell fabrication process. In addition to hole traps, electron traps were also observed with the $n^{+} p$ mesa structures. Figure 3(a) is their DLTS signal, and Fig. 3(b) their Arrhenius piots. Four electron traps could be identified at levels $E_{\xi}: E_{\mathrm{c}}-0.44 \mathrm{eV}, E_{2}: E_{\mathrm{c}}-0.62 \mathrm{eV}, E_{3}: E_{\mathrm{c}} \cdot 0.32 \mathrm{eV}$, and $E_{4}: E_{c}-0.16 \mathrm{eV}$, where $E_{\mathrm{c}}$ is the bottom of the conduction bond. The capture cross sections of these levels were calculated from their Arrhenius plots and are $\sim 10^{-21}, \sim 10^{-15}$, $\sim 10^{-19}$, and $\sim 10^{-23} \mathrm{~cm}^{2}$, respectively.

It was previously stated that the spectrum shown in Fig. 1(a) was obtained from material containing active grain boundaries. Indeed, no other regions showed the presence of hole traps, implying that all the hole (i.e., majority) traps found to exist in this material are grain-boundary related. Electron traps at large concentrations were found to exist near the bottom of the ingot and in regions close to the crucible. DLTS signals of minority traps were also obtained from regions well away from the crucible and the bottom of the ingot, and it was found that the minority trap density in such regions is much lower. This is in agreement with results of diffiusion-length measurements performed at Solarex Corp. where it was found that the minority carrier diffusion length was reduced in the lower regions of the ingot.

The main conclusion of this work is that in p-type cast polycrystalline silicon, the majority-carrier traps are mostly grain-boundary related, whereas the minority-carrier traps are found in all regions of the ingot. The minority-carrier trap concentration is larger in areas near the crucible and at the bottom of the ingot, and appears to be growth related.

This work was partiy supported by a grant from Solarex Corp. The authors would like to thank their colleagues S. M. Johnson and K. C. Yoo of Solarex Corp. for many useful discussions, and $\mathbf{S}$. Roncin for preparing the mesa structures.

\footnotetext{
${ }^{2}$ A number of papers dealing with this subject are found in Electroric and Optical Properties of Polycrystalline or Impure Semiconductors and Novel Silicon Growth Methods, edited by K. Y. Ravi and B. O'Mana (Proceed. ings of Electrochemical Society, St. Lonis, Missouri, 1980), Vol. 80-5. ${ }^{2}$ D. Y. Lang, J. Appl. Phys. 45, 3023 (1974).

3P. C. Srivastava, J. C. Bourgoin, F. Rabajo, and J. Mimila Arroyo, J. Appl. Phys. 53, 8633 (1982).

${ }^{4}$ O. S. Sastry, V. Dutta, A. K. Mukerjee, and K. L. Chopra, J. Appl. Phys. 57, $5506(1985)$.

${ }^{5}$ D. E. Iounnou, Y. J. Huang, P. K. McLarty, and S. M. Johnson, Phys. Status Solidi A 93, K223 (1986).

${ }^{6} \mathrm{H}$. J. Leamy, J. Appl. Phys. 53, R51 (1982).

${ }^{7}$ A. Broniatowski, J. Phys. (Paris) Colloq. 10, 43 (1982).

${ }^{8}$ Solitex Corp. (unpublished)
}

\title{
Characterization of free jet expansion of $\mathrm{SF}_{\mathrm{f}}$ molecules
}

\author{
V. J. Trava-Airoldi, Maria Esther Sbampato, and Alberto M. dos Santos \\ Laser Division, Instituie for Advanced Siudies, IEAv-CTA, Caixa Postal 6044, 12.231. São José dos \\ Campos, SP, Brazil \\ C. C. Ghizoni \\ Institute for Space Research, Caixa Postal 515, 12.201, São José dos Campos, SP, Brazil
}

(Received 3 September 1986; accepted ror publication 14 November 1986)

\begin{abstract}
In this work we present experimental measurements of the mean velocity of $\$ F_{6}$ molecules in a pulsed-free jet as a function of the distance from the expansion orifice. A pyroelectric detector was used to measure the time of flight of the molecules in the jet. A comparison of the experimental results with theoretical values for the mean velocity taking the temperature dependence of the specific-heat ratio into account leads to the conclusion that the freezing of rotational, vibrational, and translational temperature is reached at considerable distances from the expansion orifice.
\end{abstract}

Molecular beams have been used in many branches of scientific research. In fundamental physics they are used to measure magnetic, electric, and spin momentum in atoms and molecules. ${ }^{i}$ In chemistry, gas-kinetics and gas-dynam$i c s$, they were used to study reaction kinetics. ${ }^{1-6}$ In the beginning of this decade, molecular beams were used in photo chemical research to study the interaction of molecules and clusters with intense electromagnetic radiation fields from coherent sources. ${ }^{7-12}$

There are several conventional methods to produce a molecular beam : (a) effusive ovens which produce low-energy and low-density beams; (b) icnic sources with charge 


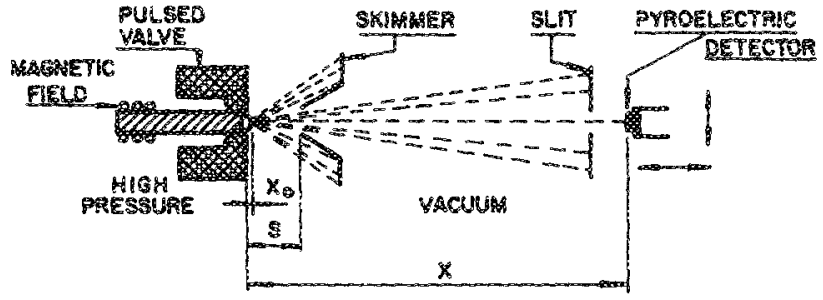

FIC. 1. Schematic of experimental setup.

exchange for highly energetic and low densiny beams; (c) shock tubes which produce pulsed beams of high energy and density; and (d) the gas-dynamic methods for production of beans of medium densities and energies.

Due to the ease of construction, wide range, and controllability of energies and densities, in addition to low translational, vibrational, and rotational temperatures obtained, the gas-dynamic methods have been largely used to study $S F_{6}$ molecules in free molecular jets. ${ }^{7-10}$

In this work, a molecular beam of $\mathrm{SF}_{6}$ molecules was produced by a supersonic expansion. The gas was pressurized to about 10 atm in a stagnation chamber made out of stainless steel and large enough as to assure constant pressure during valve operation. A puised valve, as indicated in Fig. l, with a 1-mm-diam circular orifice, connects the highpressure section to a high vacuum chamber also made out of stainless steel allowing the gas to expand during an opening time of about $80 \mu \mathrm{s}$ (FWHM). A high vacuum turbomolecular pumping system with $2000 \mathrm{l} / \mathrm{s}$ capacity was used to keep the pressure in the vacuum chamber below $10^{-5}$ mbar so that the background has no infuence on the $\$ F_{6}$ beam. Skimmer and slit are placed inside the high-vacuum section to get well collimated molecular beams. A pyroelectric detector made out of PVF 2 organic film $(6 \mu \mathrm{m}$ thick, area of 7.0 $\mathrm{mm}^{2}$ ) is placed in front of the molecular beam (Fig. 1) in a sliding mount which allows to change the distance $x$ in Fig. 1. The energy of the incident molecular jet is converted into heat at the surface of the detector ${ }^{13}$ and an electric signal is

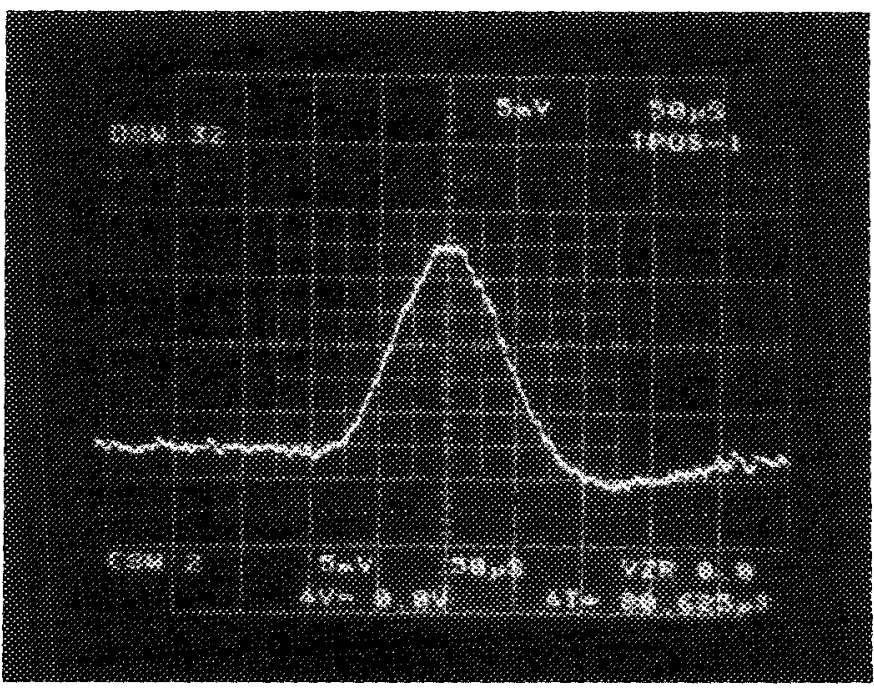

FIG. 2. Typical pulse from pyroelectric detector that shows the opening time of a pulsed valve.

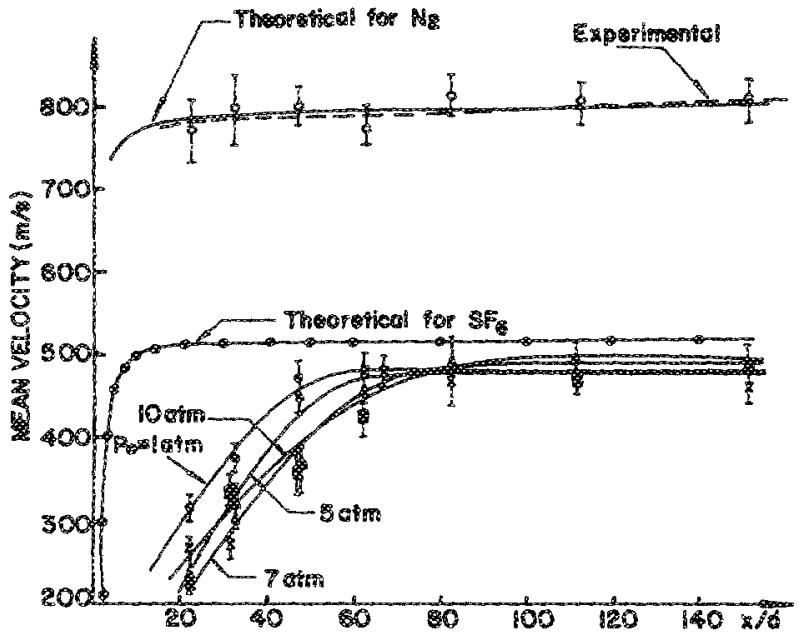

FIG. 3. The theoretical and experimental results of mean yelociry vs distance from the orifice in the center jet.

therefore generated. ${ }^{14}$ A typical pulse obtained in a screen of an oscilloscope is shown in Fig. 2. As the detector-amplifier system has a time constant of approximately $10 \mu \mathrm{s}$, the pulse width in Fig. 2 is determined by a combination of the valve opening time distribution and the spatial spread of the molecules in the jet. The mean velocity is obtained by measuring the time of fight of the molecules between the orifice and the detector. The position of the detector was changed from a distance of 11.4 to $152.0 \mathrm{~mm}$ to the expansion orifice in steps of about $10 \mathrm{~mm}$. The experimental results for the mean velocity as a function of the position are shown in Fig. 3 for different pressures in the stagnation chamber. An experiment with $\mathrm{N}_{2}$ was realized in order to calibrate TOF measurements. The results are shown in Fig. 3 , where the very good agreement with the theory validates the experimental apparatus. The theoretical curves were obtained as follows:

The translational remperature and density of the molecules in the jet are obtained from the relations ${ }^{4}$ ?

$$
T=T_{0}\left[1+(y-1) M^{2} / 2\right]^{-1}
$$

and

$$
n=n_{0}\left[1+(\gamma-1) M^{2} / 2\right\rfloor^{[1 /(\gamma-1)]},
$$

TABLE I. Dependence of the specific heat ratio on the remperature for $\mathrm{SF}_{S}$ molecules.

\begin{tabular}{cccc}
\hline$T(\mathrm{~K})$ & $\gamma$ & $T(\mathrm{~K})$ & $\gamma$ \\
\hline 20.0 & 1.333334 & 170.0 & 1.164072 \\
30.0 & 1.333328 & 180.0 & 1.153939 \\
40.0 & 1.333130 & 190.0 & 1.144994 \\
50.0 & 1.331749 & 200.0 & 1.137097 \\
60.0 & 1.327488 & 210.0 & 1.130119 \\
70.0 & 1.319118 & 220.0 & 1.123939 \\
80.0 & 1.306574 & 230.0 & 1.118454 \\
90.0 & 1.290740 & 240.0 & 1.113572 \\
100.0 & 1.272915 & 250.0 & 1.109214 \\
110.0 & 1.254367 & 260.0 & 1.105312 \\
120.0 & 1.236107 & 270.0 & 1.101808 \\
130.0 & 1.218813 & 280.0 & 1.098651 \\
140.0 & 1.202869 & 290.0 & 1.094477 \\
150.0 & 1.188438 & 300.0 & 1.093217 \\
160.0 & 1.175531 & & \\
\hline
\end{tabular}


where $M$ is the Mach number, $\gamma$ is the specific-heat ratio, and a zero subscript refers to the stagnation conditions. Here $\gamma$ and $M$ are functions of the temperature $(T)$ and consequently of the distance $x$ from the expansion orifice. Using the method of characteristics, ${ }^{15}$ a relation between $M, x$, and $y$ is written as ${ }^{4,16}$

$M=A\left(\frac{x-x_{0}}{D}\right)^{\gamma-1}-\frac{1}{2} \frac{(\gamma+1) /(\gamma-1)}{A\left[\left(x-x_{0}\right) / D\right]^{\gamma-1}}$,

with $^{4}$

$$
x_{0} / D=112.5390 \exp (-3.9196 \gamma)
$$

and

$$
\begin{aligned}
A= & 202.2092-317.7597 \gamma+21.1011 \gamma^{2} \\
& +172.2215 \gamma^{3}-69.7483 \gamma^{4}
\end{aligned}
$$

for $1.35 \leqslant \gamma \leqslant 1.05$.

The temperature dependence of $\gamma$ is obtained from the semiclassical partition function $(\mathcal{Z})$ which allows us to calculate $\gamma(T)$. We used

$$
C_{p}=-\frac{\partial}{\partial T} \frac{\partial \ln Z}{\partial(1 / K T)}
$$

to generate the data of Table I for $\mathrm{SF}_{6}$ molecules. Using the equations above and the fact that $M=1$ at $x=0$ at the orifice and for fixed stagnation conditions, $T$ and $n$ can be calculated for increasing values of the Mach number. Using an iterative process, we calculate the position $\mathrm{x}$ at which a set of compatible variables $T, \gamma, M$, and $n$ are given. From this, the theoretical curve of Fig. 3 was generated. The discrepancy between theoretical and experimental curves for the mean velocity in the region $x / d \leqslant 60$ is attributed to the fact that thermal equilibrium is not maintained in this region and so that a discharge coeficient must be considered. For $x / d>60$ region, theoretical values are greater than experimental ones because some energy is still stored in $\mathrm{SF}_{6}$ molecules as vibra- tional energy and therefore maximum theoretical translational energy is not reached. This is due to the large number of degrees of freedom of $\mathrm{SF}_{6}$ molecules. Therefore, near the orifice, relations $(1)-(5)$ are valid only for light molecules or atoms. ${ }^{4}$ The $S F_{6}$ pressure dependence of the experimental resuits is explained by the fact that the collision region increases with the pressure of the stagnation chamber resulting as a consequence of a broader region where thermal equilibrium is not maintained $(x / d<60)$. On the other hand, this pressure effect causes a higher rate of vibrational to translational energy transfer so that in the region $x / d>60$ higher molecular speeds are reached corresponding to higher stagnation pressures.

'V. B. Leonas, Sov. Phys. Usp. 7, 121 (1964).

${ }^{2}$ T. A. Milne and F. T. Greene, Advances in High Temperoture Chemistry, Vol. 2, edited by Leroy Eyring (Academic, New York, 1969), p. 107.

${ }^{3}$ V. S. Letokhov, C. B. Moore, and R. V. Ambartzumian, Chemical and Biachemical Applications of Lasers, Vol. 3, edited by C. B. Moore (Academic, New York, 1977), p. 20.

4. B. Andersen, in Gas-Dynamics, Vol. 4, Molectlar Beams, edited by P.P. Wegner (Dekker, New York, 1974), p. 1.

${ }^{5}$ D. R. Miller and R. P. Andress, J. Chem. Phys. 46, 3418 (1967).

'R. J. Gallagher and J. B. Fenn, J. Chem. Phys. 60, 3487 (1974).

'D. R. Coulter, F. R. Grabiner, L. M. Casson, G. W. Flynnand, and R. B. Bernstein, J. Chem. Phys. 73, 281 (1980).

${ }^{8}$ V. M. Apatin and G. N. Makarov, Appl. Phys. B 28, 367 (1982).

${ }^{9}$ V. M. Apatin and G. N. Makarov, Sov. Phys. JETP 57, 8 (1983).

${ }^{10}$ J. M. Zellweger, J. M. Philippoz, P. Melion, R. Monat, and H. van den Bergh, Phys. Rev. Lett. 52, 522 (1984).

"M. 1. Lester, L. M. Casson, G. B. Spector, G. W. Flynn, and R. B. Bernstein, J. Chem. Phys. 30, 1490 (1984).

${ }^{2}$ W. Radiof, V. Stert, and H. H. Ritze, Appl. Phys. B 38, 179 (1985).

${ }^{13}$ R. V. Ambartzumian, L. M. Dorozhkin, G. N. Makaror, A. A. Puretky, and B. A. Chayanov, Appl. Phys. 22, 409 (1980).

${ }^{14}$ w. B. Tiffany, Proc. Soc. Photo-Opt. Instrum. Eng. 62, 153 (1975).

${ }^{15}$ H. W. Liepmann and A. Roshko, Elements of Gasdynamics (Wiley, New York, 1957), p. 39.

16J. B. French, AIAA J. 3, 993 (1965).

\title{
Photoelectronic properties of $\mathrm{Hgl}_{2}$
}

\author{
Jonathan Bornstein and Richard H. Bube \\ Department of Materials Science and Engineering, Stanford University. Stanford, Colifornia 94305
}

(Received 13 October 1986; accepted for publication 12 December 1986)

Two types of photoelectronic measurements have been used to determine the properties of $\mathrm{HgI}_{2}$ crystals and photodetector cells: thermally stimulated conductivity, and spectral response of photoconductivity in both the lateral and transverse geometries. Characteristic trap depths are identified that compare well with previously reported values, and a decrease in apparent density with time and temperature cycling is described. Analysis of photoconductivity spectral response using the DeVore model for lateral contacts and the Goodman model for transverse contacts allows the determination of photoelectronic parameters for the material.

Mercuric iodide is a material of interest for applications to solid-state photodetection of gamma radiation in combiration with a scintillator such as calcium tungstate or thallium-activated sodium iodide. A recent symposium' has summarized much of the work in recent years. Other investi- gations have been carried out on the photoelectronic properties, ${ }^{2,3}$ transport properties, ${ }^{4}$ and photomagnetoelectric effect ${ }^{5,6}$ of $\mathrm{Hgl}_{2}$. One of the probiems with $\mathrm{HgI}_{2}$ detectors is an unexplained variation in sensitivity of the $\mathrm{HgI}_{2}$ detector with time. $^{7}$ 